\title{
DETERMINACIÓN DE LA FUNCIÓN RENAL AL FINAL DEL PERIODO DE SEGUIMIENTO EN NIÑOS DIAGNOSTICADOS DE REFLUJO VESICOURETERAL
}

\author{
Almudena Ibáñez Alonso, Mas Isabel Luis Yanes, Nayra Carmona Cedrés, Luís Antón Hernández’ y \\ Víctor García Nieto.
}

Unidades de Nefrología y Cirugía Pediátricas?. Hospital Universitario Nuestra Señora de Candelaria. Santa Cruz de Tenerife. Tenerife. España.

\begin{abstract}
Resumen.- OBJETIVO: El reflujo vesicoureteral (RVU) se ha asociado, desde antiguo, a insuficiencia renal crónica (IRC). No obstante, antes de alterarse el filtrado glomerular, pueden afectarse algunos parámetros funcionales como la osmolalidad urinaria máxima (Uosm) y la eliminación urinaria de microalbúmina.
\end{abstract}

MÉTODOS: Se han estudiado retrospectivamente las historias clínicas de 77 niños 137 varones y 40 mujeres; $48 \%$ y 52\%, respectivamente) diagnosticados de RVU y que en el momento del estudio estaban curados y tenían dos o más años de edad (6.28 3.75: rango: 2-16 años). Además de la Uosm y la eliminación urinaria de microalbúmina, se recogieron el grado de RVU, los niveles de creatinina y GFR y las anomalías morfológicas detectadas en las gammagrafías realizadas con ácido Tc-99 dimercaptosuccínico (DMSA).
RESULTADOS: Sólo cuatro pacientes tenían IRC moderada. No se observaron diferencias en los valores de Uosm según los grados de RVU. Todos los niños con RVU de grado I y II tuvieron una prueba de concentración renal normal. Se observó defecto de concentración a largo plazo en 15 niños, seis con grado III, ocho con grado IV y uno con grado V. Sólo dos pacientes con DMSA normal tuvieron la Uosm reducida. La Uosm se correlacionó directamente con el GFR ( $r=0.6$; $p<0.0011$. Con respecto a la eliminación urinaria de microaIbúmina, se comprobaron valores elevados en 11 niños, uno con grado II, cuatro con grado III y seis con grado IV. Únicamente, cuatro pacientes con DMSA normal mostraron valores de microalbúminuria por encima de lo normal. Se observó una correlación negativa entre los niveles de osmolalidad y el cociente microalbúmina/creatinina ( $r=-0.37 ; p<0.001)$. Los pacientes con cicatrices bilaterales mostraron con respecto a aquellos con DMSA normal, valores significativamente reducidos de Uosm y de GFR.

CONCLUSIONES: Al final del periodo de seguimiento, hemos comprobado defecto de la capacidad de concentración en el $19.5 \%$ y aumento de la microalbuminuria en el $14.3 \%$ de los niños que habían sido diagnosticados de RVU. La frecuencia de IRC en nuestra serie es muy reducida $(5,1 \%)$. El deterioro observado de la función renal se relaciona con la pérdida de parénquima renal más que con el grado inicial de RVU.

Palabras clave: Reflujo vesicoureteral. Nefropatía cicatrizal. Insuficiencia renal crónica. Capacidad de concentración renal. Microalbúmina. Desmopresina.

Summary.- OBJECTIVES: Vesicoureteral reflux (VUR) has been associated, since the old times, with chronic renal failure (CRF). Nevertheless, some functional parameters may be altered before glomerular filtration rate deteriorates, such as 
maximum urinary osmolality (Uosm) and urinary excretion of microalbumin.

METHODS: We retrospectively studied the records of 77 children (37 males and 14 females; $48 \%$ and $52 \%$ respectively) with the diagnosis of VUR that were cured at the time of the study and were two years old or older 16.28+- 3.75; range: 2-16 years). In addition to Uosm and urinary excretion of microalbumin, the grade of VUR, creatinine levels, GFR, and morphological anomalies detected in 99Tc dimercaptosuccinate gammagrams (DMSA) were collected.

RESULTS: Only four patients had moderate CRF. No differences in Uosm values were observed according to VUR grade. All children with grade I and II VUR had a normal renal concentration test. A long-term concentration defect was observed in 15 children, six with grade III, 8 with grade IV, and 1 with grade $V$. Only 2 patients with normal DMSA had reduced Uosm. Uosm had a direct correlation with GFR $1 r=0.6 ; p<$ 0.0011 . Regarding urinary excretion of microalbumin, elevated values were found in 11 children, one with grade II, four with grade III, and six with grade IV. Only four patients with normal DMSA showed microalbumin values over the normal range. A negative correlation between osmolality levels and microalbumin/creatinine quotient was observed $(r=-0.37$; $p<0.0011$. In comparison with patients with normal DMSA, patients with bilateral scars showed significantly lower values of Uosm and GFR.

CONCLUSIONS: At the end of the follow-up period we observed a defect on concentration capacity in $19.5 \%$ and increase of microalbuminuria in $14.3 \%$ of the children with the diagnosis of VUR. The frequency of CRF in our series is very low $(5.1 \%)$. The observed renal tubular function deterioration is more in relation with the loss of renal parenchyma than the initial grade of VUR.

Keywords: Vesicoureteral reflux. Scarring nephropathy. Renal concentration capacity. Microalbumin. Desmopressin.

\section{INTRODUCCIÓN}

Desde hace muchos años, el reflujo vesicoureteral (RVU) se ha asociado con la presencia de deterioro de la función renal, insuficiencia renal terminal y muerte (1). En el pasado, se comunicó que el RVU era la causa de insuficiencia renal crónica en el 20 a $30 \%$ de los niños y adolescentes (2). En la actualidad, esa frecuencia se ha reducido debida, principalmente, a la posibilidad de un diagnóstico más precoz y de un tratamiento más temprano de las pielonefritis que se asocian, con frecuencia, con esa malformación. No obstante, es difícil delimitar la influencia del RVU sensu stricto en la función renal puesto que pueden intervenir varios factores como el grado de RVU, la presencia de cicatrices y la asociación con otras malformaciones o con lesiones producidas intraútero, tales como la displasia re- nal (3). Esta confusión se percibe mejor si se considera que durante muchos años, la nefropatía cicatrizal se denominó nefropatía de reflujo (4) y, antes, pielonefritis crónica (5).

Hemos estudiado la función renal de un grupo de niños diagnosticados de RVU al final del periodo de seguimiento, una vez que el RVU ya se había curado, bien con tratamiento médico o bien, con tratamiento quirúrgico. Además de la función glomerular renal (GFR), se recogieron otros parámetros funcionales más sensibles como la osmolalidad urinaria máxima y la eliminación urinaria de microalbumina (6).

\section{PACIENTES Y MÉTODOS}

Se han estudiado retrospectivamente las historias clínicas de 77 niños (37 varones y 40 mujeres; $48 \%$ y $52 \%$, respectivamente) diagnosticados de RVU que en el momento del estudio estaban curados y tenían dos o más años de edad (6.28 3 3.75: rango: 2-16 años).

El $50,9 \%$ de los casos habían sido curados con tratamiento cistoscópico, el 32,1\% con tratamiento médico y el $17 \%$ con tratamiento quirúrgico.

Para clasificar el grado de reflujo, se utilizó la clasificación internacional que cataloga la malformación en cinco grados en función del nivel alcanzado y del grado de dilatación del sistema colector (7). Si el reflujo era bilateral, para la clasificación se consideraba el de mayor grado.

Las anomalías morfológicas asociadas se estudiaron mediante la realización de una gammagrafía con 99mTc-ácido dimercaptosuccínico (DMSA). El estudio se efectúa a las tres o cuatro horas de la inyección en varias proyecciones y permite, a la vez, una valoración morfológica del parénquima renal y una medida de la función renal porcentual de cada riñón.

El cálculo del GFR se realizó según la fórmula de Schwartz. Para ello, se utilizan los siguientes valores para la constante K: 0.55 en niños a partir del año de vida hasta la adolescencia, 0.57 en mujeres adolescentes y 0.70 en varones adolescentes $(8,9)$.

La capacidad de concentración renal se determinó tras la administración de $20 \mu \mathrm{g} \circ 0.2 \mathrm{mg}$. de desmopresina (DDAVP), intranasal u oral, respectivamente. No se restringió la ingesta de líquidos durante la prueba. Se recogieron tres muestras de orina durante intervalos de $90 \mathrm{mi}-$ nutos, dándose como resultado de la prueba el valor mayor obtenido en cualquiera de las tres muestras. Se considera que el límite inferior de la normalidad de la osmolalidad urinaria máxima por encima del año de edad es de 835 $\mathrm{mOsm} / \mathrm{kg}(10-11)$.

La eliminación urinaria de microalbúmina (MAU) se estableció mediante el cálculo del cociente entre las concentraciones de MAU y creatinina cuantificados en una muestra correspondiente a la primera orina del día. Se consideran valores normales del cociente aquellos inferiores a $2.3 \mu \mathrm{g} / \mu \mathrm{mol}$ (12). 
La creatinina sérica se determinó con el método de la creatininasa utilizando un autoanalizador Modular Analytics (Roche/Hitachi, Mannheim, Alemania). La osmolalidad urinaria se cuantificó tras la determinación de la depresión del punto de congelación en un osmómetro Osmo Station OM-6050 (Menarini Diagnostics, Florencia, Italia). La microalbúmina se midió mediante una técnica nefelométrica (Array).

Los datos obtenidos fueron procesados con el programa de estadística SPSS 13.0. Las variables cuantitativas se expresaron como media y desviación estándar. El test de la U de Mann-Whitney se utilizó para comparar variables cuantitativas independientes. La correlación entre diferentes variables se realizó usando el test de la " $r$ " de Pearson. Un valor de probabilidad menor de 0.05 fue considerado estadísticamente significativo.

\section{RESULTADOS}

Sólo cuatro pacientes tenían insuficiencia renal crónica moderada $(5,1 \%)$. Sus valores de GFR eran 60.5, $52.7,68.4$ y $71.30 \mathrm{ml} / \mathrm{min} / 1.73 \mathrm{~m}^{2}$, respectivamente. En estos cuatro niños, los valores de osmolalidad urinaria máxima fueron 441, 338, 238 y $269 \mathrm{mOsm} / \mathrm{kg}$, respectivamente. Los tres primeros habían tenido RVU grado IV y mostraban cicatrices bilaterales en el DMSA, mientras que el último tuvo un reflujo grado $\mathrm{V} y$ era portador de varias cicatrices unilaterales en el DMSA.

No se apreciaron diferencias significativas en los valores de osmolalidad urinaria máxima, de GFR y de la eliminación urinaria de microalbúmina al clasificar a los pacientes según el grado de RVU que habían tenido al diagnóstico (Tabla I).

Se observó defecto de concentración, a largo plazo, en 15 niños (19.5\%), 6 con grado III, 8 con grado IV y
1 con grado $V$. Todos los niños que habían sido diagnosticados de RVU grados I y II mostraron valores normales en la prueba de concentración urinaria, al final del periodo de seguimiento.

Además, sólo dos pacientes con DMSA normal obtuvieron una osmolalidad urinaria máxima reducida. Ésta, estaba disminuida en el 29.4\% (5/17) de los niños con riñón atrófico unilateral o ausente y en el $83.3 \%(5 / 6)$ de aquellos con cicatrices bilaterales. Los niños restantes con defecto de concentración eran portadores de una cicatriz o de varias cicatrices unilaterales.

Con respecto a la eliminación urinaria de microalbúmina, existió alteración del cociente $\mathrm{MAU} / \mathrm{Cr}$ en 11 niños (14.3\%), uno con RVU grado II, cuatro con RVU grado III y seis con RVU grado IV.

Sólo cuatro pacientes con DMSA normal mostraron valores de microalbúminuria por encima del límite alto de la normalidad. Ésa, estaba aumentada en el $11.7 \%$ $(2 / 17)$ de los niños con riñón atrófico unilateral o ausente y en el $40 \%(2 / 5)$ de aquellos con cicatrices bilaterales. Los niños restantes eran portadores de una cicatriz o de varias unilaterales.

Las alteraciones más pronunciadas se dieron en aquellos pacientes con menor parénquima renal funcionante Los pacientes con cicatrices bilaterales mostraron con respecto a aquellos con parénquima renal normal, valores significativamente reducidos de Uosm y de GFR y significativamente elevados de los niveles de creatinina (Tabla II).

En el conjunto de la muestra, se comprobó una relación directamente proporcional entre la osmolalidad urinaria máxima y el GFR ( $r=0.6 ; p<0.001)$ (Figura 1), así como una correlación negativa entre los niveles de osmolalidad y el cociente microalbúmina/creatinina $(r=-0.37$; $\mathrm{p}<0.001$ ) (Figura 2).

\section{TABLA I. VALORES DE OSMOLALIDAD URINARIA MÁXIMA, ELIMINACIÓN URINARIA DE MICROALBÚMINA Y GFR AL DIVIDIR A LOS PACIENTES EN FUNCIÓN DEL GRADO DE RVU.}

\begin{tabular}{|c|c|c|c|c|}
\hline Grado de RVU & $\begin{array}{c}\text { Osmolalidad } \\
\text { urinaria máxima } \\
\text { (mOsm/kg) }\end{array}$ & $\begin{array}{l}\text { Microalbúmina/ } \\
\text { creatinina } \\
\text { (pg/pmol) }\end{array}$ & $\begin{array}{c}\text { GFR } \\
\left(\mathrm{ml} / \mathrm{min} / 1,73 \mathrm{~m}^{2}\right)\end{array}$ & $\begin{array}{c}\text { Creatinina } \\
\text { (mg/dl) }\end{array}$ \\
\hline I $(n=2)$ & $984,5 \pm 154,8$ & $1,34 \pm 0,41$ & $144,7 \pm 9,3$ & $0,38 \pm 0,1$ \\
\hline$\|(n=15)$ & $969,3 \pm 93,8$ & $1,38 \pm 0,64$ & $147,4 \pm 31,7$ & $0,47 \pm 0,11$ \\
\hline III $(n=32)$ & $937,5 \pm 119,3$ & $1,32 \pm 0,78$ & $145,1 \pm 30,1$ & $0,46 \pm 0,11$ \\
\hline IV $(n=26)$ & $866,9 \pm 183,3$ & $3,81 \pm 7,59$ & $131,5 \pm 41,2$ & $0,56 \pm 0,25$ \\
\hline$V(n=2)$ & $835,5 \pm 99,7$ & $0,41 \pm 0,14$ & $120,9 \pm 8,1$ & $0,49 \pm 0,02$ \\
\hline
\end{tabular}




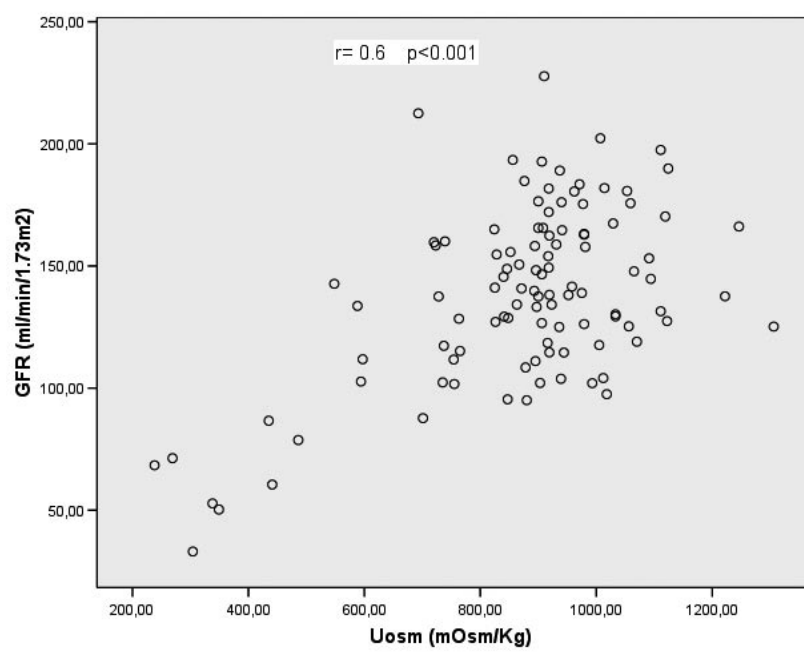

FIGURA 1. Relación entre la osmolalidad urinaria máxima (Uosm) y el GFR.

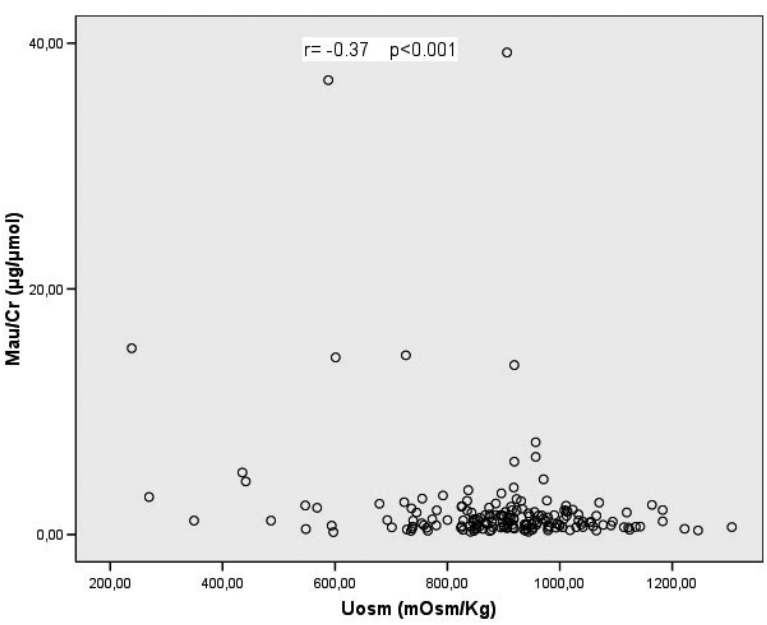

FIGURA 2. Relación entre la osmolalidad urinaria máxima (Uosm) y el cociente microalbúmina/creatinina (MAU/Cr).

\section{DISCUSIÓN}

La presencia de RVU no sólo se ha asociado, a largo plazo, con reducción del GFR e insuficiencia renal crónica $(2,13-16)$. También, se ha descrito que los niños con RVU pueden tener diversos trastornos de la función tubular renal como defecto de la capacidad de concentración (17-22), incremento en la eliminación urinaria de $\mathrm{N}$-acetilglucosaminidasa (23-28) y de otras proteínas que se consideran marcadores de la función tubular proximal $(25,28)$, así como defecto en la capacidad de acidificación renal $(29,30)$.

En nuestra serie, la anomalía funcional más frecuente fue el defecto en la capacidad de concentración.
Nosotros, hemos descrito previamente que esta prueba es la más sensible para detectar reducción del parénquima renal (6). Observamos una reducción de la osmolalidad urinaria máxima en el 19,5\% de los niños. Este defecto, se relaciona especialmente con la pérdida de parénquima renal observada en el DMSA más que con el grado de RVU.

Esta observación ya había sido comunicada por Walter y cols. (19), en 1983, los cuales apreciaron que un defecto persistente en la capacidad de concentración renal es predictor de la presencia de cicatrices renales y de un pobre crecimiento renal.

\section{TABLA II. VALORES DE OSMOLALIDAD URINARIA MÁXIMA, ELIMINACIÓN URINARIA DE MICROALBÚMINA Y GFR AL DIVIDIR A LOS PACIENTES EN FUNCIÓN DE LAS LESIONES MORFOLÓGICAS DETECTADAS EN LA GAMMAGRAFÍA REALIZADA CON DMSA.}

\begin{tabular}{|c|c|c|c|c|}
\hline DMSA & $\begin{array}{c}\text { Osmolalidad } \\
\text { urinaria máxima } \\
\text { (mOsm/kg) }\end{array}$ & $\begin{array}{c}\text { Microalbúmina/ } \\
\text { creatinina } \\
\text { (pg/pmol) }\end{array}$ & $\begin{array}{c}\text { GFR } \\
\left(\mathrm{ml} / \mathrm{min} / 1,73 \mathrm{~m}^{2}\right)\end{array}$ & $\begin{array}{l}\text { Creatinina } \\
\text { (mg/dl) }\end{array}$ \\
\hline Una cicatriz $(n=14)$ & $967,1 \pm 108,4$ & $1,45 \pm 0,78$ & $159,5 \pm 31,7$ & $0,45 \pm 0,14$ \\
\hline Varias cicatrices unilaterales $(n=12)$ & $896,6 \pm 81$ & $1,38 \pm 1,55$ & $138,7 \pm 15,7$ & $0,48 \pm 0,91$ \\
\hline Cicatrices bilaterales $(n=6)$ & $620 \pm 210^{*}$ & $8,56 \pm 16$ & $98,5 \pm 39,9 * *$ & $0,73 \pm 0,28 * * *$ \\
\hline Riñón atrófico, displásico o ausente $(n=17)$ & $920,58 \pm 105$ & $1,64 \pm 1,84$ & $141,2 \pm 24,3$ & $0,49 \pm 0,16$ \\
\hline Parénquima renal normal $(n=28)$ & $971,08 \pm 116,15$ & $2,03 \pm 2,76$ & $141,75 \pm 32,11$ & $0,49 \pm 0,14$ \\
\hline
\end{tabular}


La reducción de parénquima renal producida por la presencia de cicatrices y por la displasia renal que se asocian con RVU, produce hiperfiltración en el parénquima renal restante. Como consecuencia, en estos casos, puede observarse un incremento en la eliminación urinaria de microalbúmina. Este parámetro no ha sido excesivamente estudiado en niños con RVU $(25,28,31-34)$. En nuestra serie, como en el caso de la osmolalidad, la presencia de alteraciones en la cuantificación de la microalbúmina urinaria la relacionamos directamente con las anomalías morfológicas detectadas en el DMSA.

Como corolario de lo expuesto, los niños con cicatrices bilaterales mostraron un mayor deterioro de la función renal (Tabla II). En contra, los niños portadores de un riñón único, aunque morfológicamente normal, gracias a la hipertrofia compensadora propia de estos casos, no mostraron diferencias en los parámetros estudiados de función renal con respecto a los niños con riñones con parénquima renal normal (Tabla II).

Es de destacar el que no observáramos diferencias significativas en los parámetros de función renal al clasificar a los pacientes de acuerdo con el grado inicial de reflujo (Tabla I). La razón debe buscarse en que, en la mayoría de los casos diagnosticados en la infancia, la hiperpresión asociada al RVU, va reduciéndose progresivamente. De ahí, el que en muchos casos se contemple una curación espontánea con el paso del tiempo.

En nuestra serie, hemos comprobado, únicamente, la presencia de insuficiencia renal crónica moderada en el $5,1 \%$ de los casos. Esto implica, obviamente, que han mejorado las condiciones diagnósticas y terapéuticas de este tipo de pacientes con respecto al pasado, en el que se describió una alta frecuencia de niños y adolescentes que acababan en insuficiencia renal crónica terminal. Sin ahondar en el tema, esta mejoría, debe relacionarse con las condiciones sanitarias de nuestro país y con la posibilidad de contar con mejores y más eficaces métodos diagnósticos y terapéuticos.

En cuanto a las condiciones sanitarias, deben remarcarse la disponibilidad de un sistema sanitario abierto, prácticamente, al $100 \%$ de la población, la existencia de pediatras en Centros de Salud en cualquier punto de nuestra geografía y la disponibilidad de cirujanos pediátricos dedicados a urología en los hospitales pediátricos de tercer nivel. Con respecto a las mejoras diagnósticas, ha sido definitiva la posibilidad del diagnóstico intraútero de los recién nacidos con dilatación de las vías urinarias. En fin, un diagnóstico precoz de las pielonefritis y la instauración de un tratamiento antibiótico precoz y adecuado permite la formación de un menor número de cicatrices y con una menor extensión.

\section{BIBLIOGRAFÍA Y LECTURAS RECOMENDADAS (*lectura de interés $y^{* *}$ lectura fundamental)}

1. STEELE, R.E.; LEADBETTER, G.W.; CRAWFORD, J.D.: "Prognosis of childhood urinary-tract infection. The current status of patients hospitalised between 1940 and 1950”. N. Engl. J. Med., 269: 883, 1963.

2. BROYER, M.; DONCKERWOLCKE, R.A.; BRUNNER, F.P. y cols.:, "Combined report on regular dialysis and transplantation of children in Europe, 1980". Proc. Eur. Dial. Transplant. Assoc., 18: 60, 1981.

3. SCOTT, J.E.S.: "Fetal ureteric reflux". Br. J. Urol., 59: 291, 1987.

4. HODSON, C.J.; EDWARDS, D.: "Chronic pyelonephritis and vesico-ureteric reflux". Clin. Radiol., 11: 219, 1960.

5. BAILEY, R.R.: "The relationship of vesico-ureteric reflux to urinary tract infection and chronic pyelonephritis-reflux nephropathy". Clin. Nephrol., 1: 132, 1973.

*6. GARCÍA NIETO, V.; LUIS YANES, M.I.; HERNÁNDEZ GONZÁLEZ, M.J. y cols.: "Sensitivity and specificity of four procedures for estimating the renal function to detect morphological anomalies in paediatric patients". Pediatr. Nephrol., 20: 104, 2005.

7. MEDICAL VERSUS SURGICAL TREATMENT OF PRIMARY VESICOURETERAL REFLUX: "Report of the International Reflux Study Committee". Pediatrics, 67: 392, 1981.

8. SCHWARTZ, G.J.; HAYCOCK, G.B.; EDELMANN, C.M. Jr. y cols.: "A simple estimate of glomerular filtration rate in children derived from body length and plasma creatinine”. Pediatrics, 58: 259, 1976.

9. SCHWARTZ, G.J.; GAUTHIER, B.: “A simple estimate of glomerular filtration rate in adolescent boys". J. Pediatr., 106: 522, 1985.

10. MONNENS, L.; SMULDERS, Y.; VAN LIER, H. y cols.: "DDAVP test for assessment of renal concentrating capacity in infants and children". Nephron., 29: $151,1981$.

11. SANTOS, F.; GARCÍA NIETO, V.: "Exploración basal de la función renal". García-Nieto V, Santos F, Rodríguez-Iturbe B, eds. Nefrología Pediátrica, $2^{\mathrm{a}}$ ed. Madrid: Aula Médica, 2006.

12. GIBB, D.M.; SHAH, V.; PREECE, M. y cols.: "Variability of urine albumin excretion in normal and diabetic children". Pediatr. Nephrol., 3: 414, 1989.

13. APERIA, A.; BROBERGER, O.; ERICSSON, N.O. y cols.: "Effect of vesicoureteral reflux on renal function in children with recurrent urinary tract infections". Kidney Int., 9: 418, 1976.

14. MUNDY, A.R.; KINDER, C.H.; JOYCE, M.R. y cols.: "Improvement in renal function following ureteric reimplantation for vesicoureteric reflux". Br. J. Urol., 53: 542, 1981.

15. BERG, U.B.: "Long-term followup of renal morphology and function in children with recurrent pyelonephritis". J. Urol., 148: 1715, 1992.

16. MINDRUP, S.R.; COOPER, C.S.; HODROFF, M.A. y cols.: "Comparison of relative renal function by renal scintigraphy and lateralized creatinine clearance in children with bilateral vesicoureteral reflux". Urology, 61: 816, 2003. 
*17. UEHLING, D.T.: "Effect of vesicoureteral reflux on concentrating ability". J. Urol., 106: 947, 1971.

18. WALKER, D.; RICHARD, G.; DOBSON, D. y cols.: "Maximum urine concentration. Early means of identifying patients with reflux who may require surgery". Urology, 1: 343, 1973.

**19. WALKER, R.D. 3rd.; RICHARD, G.A.; FENNELL, R.S. y cols.: "Renal growth and scarring in kidneys with reflux and a concentrating defect". J. Urol., 129: 784, 1983.

20. KEKOMÄKI, M.; WALKER, R.D.: "Fractional excretion of magnesium and renal concentrating capacity in refluxing renal units". J. Urol., 140: 1095, 1983.

21. GARCÍA NIETO, V.; ERICE KEPPLER, M.L.; GARCÍA BÁEZ, M. y cols.: "Alteración de la capacidad de concentración renal como expresión de lesión tubular en el reflujo vesicoureteral en la infancia". An. Esp. Pediatr., 31: 498, 1989.

22. GOBET, R.; CISEK, L.J.; CHANG, B. y cols.: "Experimental fetal vesicoureteral reflux induces renal tubular and glomerular damage, and is associated with persistent bladder instability". J. Urol., 162: 1090, 1999.

23. TAKEDA, M.; KATAYAMA, Y.; TAKAHASHI, H. y cols.: "Study on urinary low molecular protein and enzyme in primary vesico-ureteral reflux patients". Nippon Hinyokika Gakkai Zasshi, 81: 454, 1990.

24. CARR, M.C.; PETERS, C.A.; RETIK, A.B. y cols.: "Urinary levels of renal tubular enzyme N-acetyl- $\beta$-Dglucosaminidase in relation to grade of vesicoureteral reflux". J. Urol., 146: 654, 1991.

25. KOMEYAMA, T.; TAKEDA, M.; KATAYAMA, Y. y cols.: "Value of urinary endothelin-1 in patients with primary vesicoureteral reflux". Nephron, 65: 537, 1993.
26. MIYAKITA, H.; PURI, P.: "Urinary levels of N-acetylbeta-D-glucosaminidase: A simple marker for predicting tubular damage in higher grades of vesicoureteric reflux". Eur. Urol., 25: 135, 1994.

27. TOMLINSON, P.A.; SMELLIE, J.M.; PRESCOD, N. y cols.: "Differential excretion of urinary proteins in children with vesicoureteric reflux and reflux nephropathy". Pediatr. Nephrol., 8: 21, 1994.

*28. KONDA, R.; SAKAI, K.; OTA, S. y cols.: "Follow up study of renal function in children with reflux nephropathy after resolution of vesicoureteral reflux". J. Urol., 157: 975, 1997.

29. GUIZAR, J.M.; KORNHAUSER, C.; MALACARA, J.M. y cols.: "Renal tubular acidosis in children with vesicoureteral reflux". J. Urol., 156: 193, 1996.

30. SHARMA, R.K.; JHA, R.; BHATIA, V.L. y cols.: "Secondary distal renal tubular acidosis in association with urological abnormalities". Nephrol Dial Transplant, 12: 233, 1997.

31. SAKAI, K.; KONDA, R.; ORIKASA, S. y cols.: "Studies on reflux nephropathy. Renal tubular and glomerular damage evaluated by various urinary indices". Nippon Hinyokika Gakkai Zasshi, 84: 364, 1993.

32. KONDA, R.; SAKAI, K.; OTA, S. y cols.: "Urinary excretion of epidermal growth factor in children with reflux nephropathy". J. Urol., 157: 2282, 1997.

33. LAMA, G.; SALSANO, M.E.; PEDULLA, M. y cols.: "Angiotensin converting enzyme inhibitors and reflux nephropathy: 2-year follow-up". Pediatr. Nephrol., 11: 714, 1997.

**34. LAMA, G.; TEDESCO, M.A.; GRAZIANO, L. y cols.: "Reflux nephropathy and hypertension: Correlation with the progression of renal damage". Pediatr. Nephrol., 18: 241, 2003. 